\title{
Unimodal Responses Prevail within the Multisensory Claustrum
}

\author{
Ryan Remedios, ${ }^{1}$ Nikos K. Logothetis, ${ }^{1,2}$ and Christoph Kayser ${ }^{1}$ \\ ${ }^{1}$ Max Planck Institute for Biological Cybernetics, 72076 Tübingen, Germany, and 2Division of Imaging Science and Biomedical Engineering, University of \\ Manchester, Manchester M13 9PT, United Kingdom
}

The claustrum receives afferent inputs from multiple sensory-related brain areas, prompting speculation about a role in integrating information across sensory modalities. Here we directly test this hypothesis by probing neurons in the primate claustrum for functional characteristics of multisensory processing. To this end we recorded neuronal responses to naturalistic audio-visual stimuli from the claustra of alert monkeys. Our results reveal the existence of distinct claustral zones comprised of unimodal neurons associated with the auditory and visual modalities. In a visual zone within the ventral claustrum neurons responded to visual stimuli but not to sounds, whereas in an auditory zone located within the central claustrum neurons responded to sounds but not to visual stimuli. Importantly, we find that neurons within either zone are not influenced by stimuli in the other modality and do not exhibit the typical response characteristics usually associated with multisensory processing. While these results confirm the notion of the claustrum as multisensory structure per se, they argue against the hypothesis of the claustrum serving as an integrator of sensory information.

\section{Introduction}

While the exact function of the claustrum remains speculative, recent studies have assigned an integrative role to it. More specifically, the claustrum was hypothesized to play a role in integrating sensory information across different modalities (Sherk, 1986; Ettlinger and Wilson, 1990; Edelstein and Denaro, 2004; Crick and Koch, 2005). This hypothesis was promoted by a combination of indirect anatomical and functional evidence. First, anatomical studies revealed afferent connections from various cortical and subcortical sensory structures (Pearson et al., 1982; TannéGariépy et al., 2002; Fernández-Miranda et al., 2008) and reported an apparent topographical mapping of the neocortex onto the claustrum (Pearson et al., 1982; Sherk, 1986; FernándezMiranda et al., 2008). Second, electrophysiological studies reported claustral neurons that responded to stimuli in the visual, acoustic, and somatic modalities (Segundo and Machne, 1955; Spector et al., 1970, 1974; Olson and Graybiel, 1980; LeVay and Sherk, 1981; Sherk and LeVay, 1981; Clarey and Irvine, 1986). In analogy to other brain structures that receive similar diverse sensory afferents and indeed participate in sensory integration, these observations were taken as evidence supporting the integrative function of the claustrum (Sherk, 1986; Ettlinger and Wilson, 1990; Edelstein and Denaro, 2004; Crick and Koch, 2005). However, and despite some early observations, direct tests for a neural correlate of sensory integration in the claustrum have been missing.

Received June 8, 2010; revised July 17, 2010; accepted July 26, 2010.

This work was supported by the Max Planck Society and the German Research Foundation (KA 2661/1).

Correspondence should be addressed to Christoph Kayser, Max Planck Institute for Biological Cybernetics,

Spemannstrasse 38, 72076 Tübingen, Germany. E-mail: christoph.kayser@tuebingen.mpg.de.

DOI:10.1523/JNEUROSCI.2937-10.2010

Copyright $\odot 2010$ the authors $\quad 0270-6474 / 10 / 3012902-06 \$ 15.00 / 0$
In part, this lack of knowledge about the claustrum's function is due to its complex morphology and proximity to the insular cortex. In primates, the claustrum comprises a thin sheet of cells situated close to the insula and surrounded by the white matter of the external capsule medially and the extreme capsule laterally. Its shape narrows dorsally and thickens ventrally and appears to wrap around the dorsoventral extent of the insular cortex, hence exhibiting a crooked morphology. This intricate shape makes it difficult to separate claustral from insula activations in functional imaging studies and renders evidence from such methodologies indecisive (Hadjikhani and Roland, 1998; Banati et al., 2000; Naghavi et al., 2007). In addition, this shape also poses considerable challenges for electrophysiological or microstimulation approaches to this structure. As a consequence, many of the earlier studies on the claustrum were conducted on anesthetized animals, and most were based on the relatively expanded and more accessible dorsal zone of the claustrum in the cat (Segundo and Machne, 1955; Spector et al., 1970, 1974; Olson and Graybiel, 1980; LeVay and Sherk, 1981; Sherk and LeVay, 1981; Clarey and Irvine, 1986; Cortimiglia et al., 1991). This not only leaves large portions of this structure unstudied, but also makes extrapolation to alert animals and to the primate brain difficult.

In this study we directly tested the hypothesis that claustral neurons participate in integrating stimuli across the sensory modalities. In particular, we recorded neural responses in the claustrum of alert nonhuman primates to audio-visual stimuli and used established criteria to probe individual neurons for functional characteristics of multisensory processing and integration.

\section{Materials and Methods}

Two adult male rhesus monkeys (Macaca mulatta) participated in these experiments. All procedures were approved by the local authorities (Regierungspräsidium) and were in full compliance with the guidelines of 

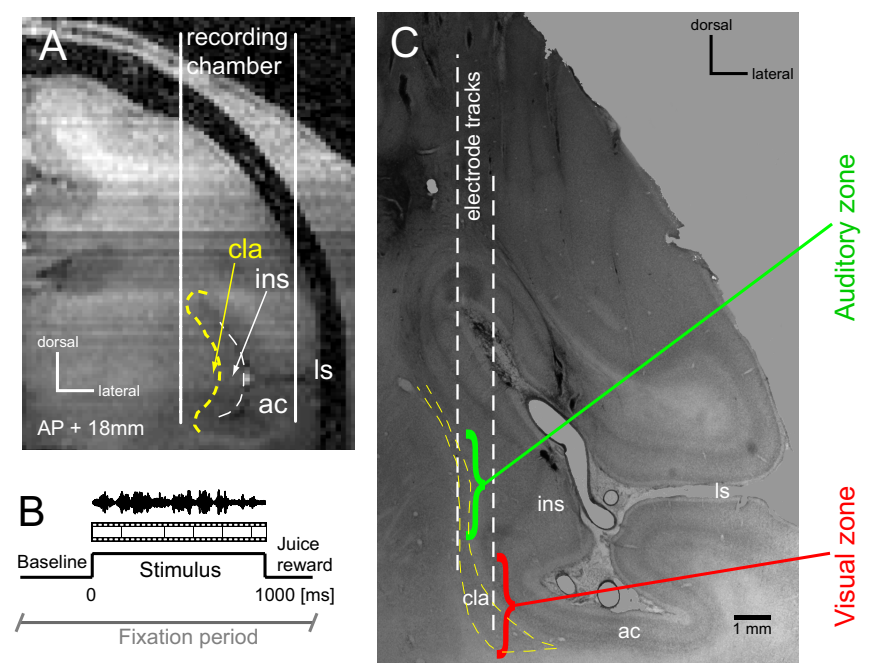

D
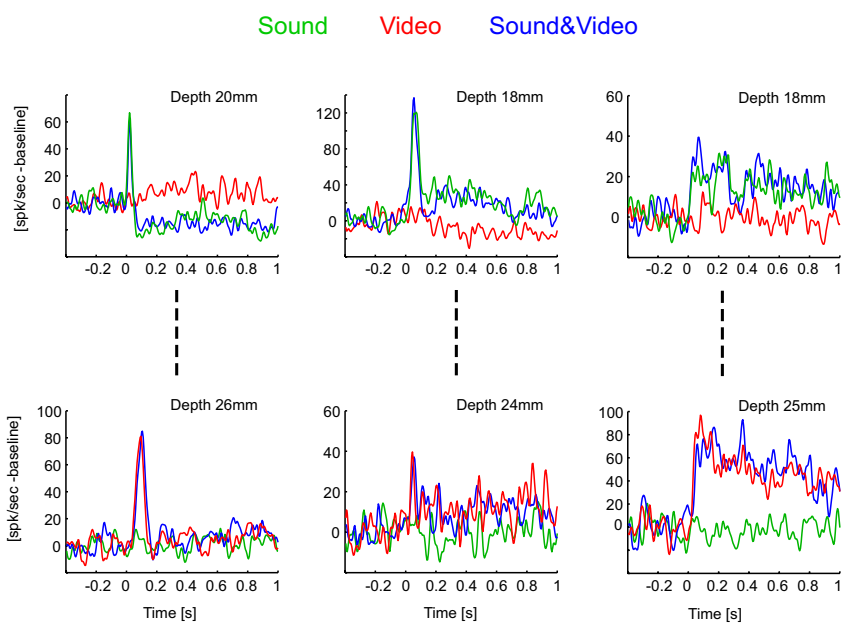

Figure 1. Auditory and visual zones in the claustrum. $\boldsymbol{A}$, Illustration of the vertical approach to the claustrum used in one animal, depicted on an anatomical MR image. $\boldsymbol{B}$, Timeline of the audio-visual stimulation paradigm. $C$, Nissl-stained section used to confirm the approach to the claustrum using visible electrode tracks. Approximate locations of the auditory and visual zones are indicated. $\boldsymbol{D}$, Example data recorded on three penetrations demonstrating the subsequent occurrence of auditory and visually driven units at different depths from the cortical surface. Lines denote the trial-averaged firing rate to visual (video), auditory (sound) and combined audio-visual (sound\&video) stimuli (smoothed using a 10 ms Gaussian window); baseline firing rates were subtracted. Stimuli commenced at $t=0$. cla, claustrum; ins, insular cortex; ac, auditory cortex; Is, lateral sulcus.

the European Community (EUVD 86/609/EEC) for the care and use of laboratory animals. All surgical procedures were performed under aseptic and sterile conditions. Briefly, neural responses were recorded using a custom-made multielectrode system, from alert animals subjected to auditory and visual stimuli. Signals were amplified using an Alpha Omega system (Alpha Omega $\mathrm{GmbH}$ ), filtered between $4 \mathrm{~Hz}$ and $9 \mathrm{kHz}$ and digitized at $20.83 \mathrm{kHz}$. The general procedures used in this study have been previously published (Kayser et al., 2008; Remedios et al., 2009; Dahl et al., 2010).

To approach the claustrum, recording chambers were positioned based on preoperative magnetic resonance (MR) images and stereotaxic coordinates. In one animal the claustrum was targeted at an angle of $20^{\circ}$ anteroposterior (AP) and $45^{\circ}$ dorsoventral (DV) so that recordings were centered at approximately AP $+14 \mathrm{~mm}, \mathrm{DV}+18 \mathrm{~mm}$. In the other animal the claustrum was approached vertically, with recordings centered at approximately AP $+18 \mathrm{~mm}, \mathrm{DV}+17 \mathrm{~mm}$ (Fig. $1 \mathrm{~A}$ ).

Neural activity was recorded in response to an audio-visual stimulation paradigm while the animals performed a visual fixation task (Fig. $1 B$ ). Naturalistic $1 \mathrm{~s}$ long auditory (sound only), visual (video only) and bimodal audio-visual stimuli (sound\&video) were presented in pseudorandom sequence with an intertrial interval of $2-2.5 \mathrm{~s}$. These stimuli comprised videos and sounds of behaviorally relevant scenes, such as movies of vocalizing conspecifics or other animals in their natural settings (see Kayser et al., 2008; Remedios et al., 2009; Dahl et al., 2010, for details). Sounds were presented from two calibrated free field speakers (JBL Professional, positioned $70 \mathrm{~cm}$ from the head and $50^{\circ}$ to left and right) at an average intensity of $65 \mathrm{~dB}$ SPL. Visual stimuli were presented on a 21 inch gamma-corrected monitor and covered a visual field of $24 \times 18^{\circ}$.

The data were analyzed in Matlab (MathWorks). Spike-sorted activity was extracted using commercial spike-sorting software (Plexon Offline Sorter) after high-pass filtering the raw signal at $500 \mathrm{~Hz}$. For the present analysis we did not distinguish between single and multiunit sites. Significant responses of individual units to sensory stimuli were determined using a $z$-score, which quantifies the response amplitude relative to the variability during the prestimulus baseline period. We considered only units for which the response to at least one sensory modality breached a threshold of $3 \mathrm{SD}$. This resulted in the inclusion of 128 responsive neurons from animal I05 and 63 neurons from animal M03. The first time bin at which the averaged response exceeded two SD from baseline for at least 10 consecutive milliseconds was considered the "response onset latency" and reported as the median, $25^{\text {th }}$ and $75^{\text {th }}$ percentiles (Remedios et al., 2009). Multisensory response properties were subsequently characterized following established criteria (Kayser et al., 2008; Dahl et al., 2009). The "response amplitude" for each unit and modality condition was computed by first finding the peak of the trial averaged response and then computing (for each trial) the mean spike count in a $100 \mathrm{~ms}$ time window centered on this peak. For further analysis (except where noted) these response amplitudes were corrected for differences in baseline activity by subtracting (for each trial) the spike-count in a $100 \mathrm{~ms}$ window during the prestimulus baseline period. To determine the "modality preference" of each unit, responses to auditory and visual stimuli were compared using a Wilcoxon rank-sum test, and units with a significant $(p<0.05)$ difference were labeled by the modality eliciting the stronger response. "Bimodal" neurons were defined as neurons responding with (significant) deviations from baseline to both visual and acoustic stimuli. To this end we directly compared the (non-baseline corrected) activity during presentation of stimuli in the nonpreferred modality to the prestimulus baseline activity (Wilcoxon rank-sum test). Finally, neurons were tested for typical characteristics of sensory "integration" by examining whether the response to bimodal stimuli deviates from the response expected by a trivial (linear) superposition of the responses to unimodal auditory and visual stimuli (Stein and Meredith, 1993). Practically this test was performed using an established bootstrap procedure (Stanford et al., 2005). Local field potentials (LFP) were defined by lowpass filtering $(120 \mathrm{~Hz})$ the raw data. The LFP response amplitude was obtained from the (stimulus-averaged) evoked response by summing the total LFP deflection in a time interval of $0-200 \mathrm{~ms}$ following stimulus onset. Response amplitudes to visual and auditory stimuli were compared using a Wilcoxon signed-rank test.

\section{Results}

The claustrum comprises a thin sheet of cells, situated close to the insular cortex. Our strategy to sample neurons from the claustrum was based on a combination of anatomical and functional landmarks. First, anatomical MR images were used to calculate the position of the claustrum relative to the recording chamber (Fig. 1A). The correctness of this was confirmed postmortem in Nissl-stained sections of brain tissue (Fig. 1C). Second, responses of neurons in neighboring structures such as the insular cortex were characterized as part of previous studies (Remedios et al., 2009), and were used to functionally differentiate claustrum and insula. While insula neurons respond to longer natural sounds 
A

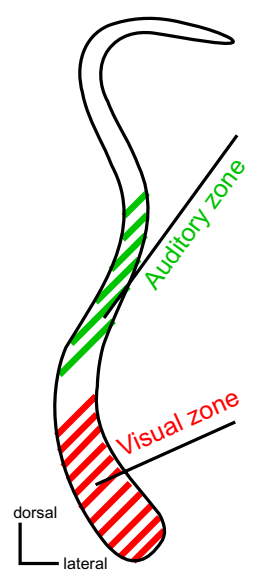

B
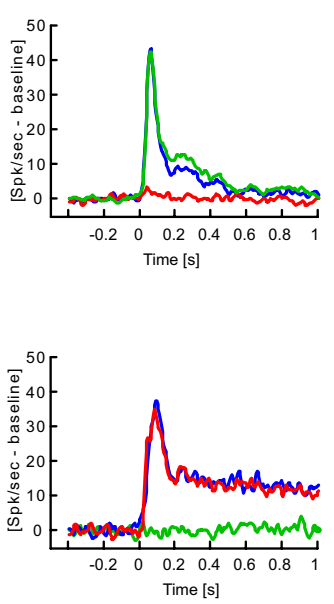

C
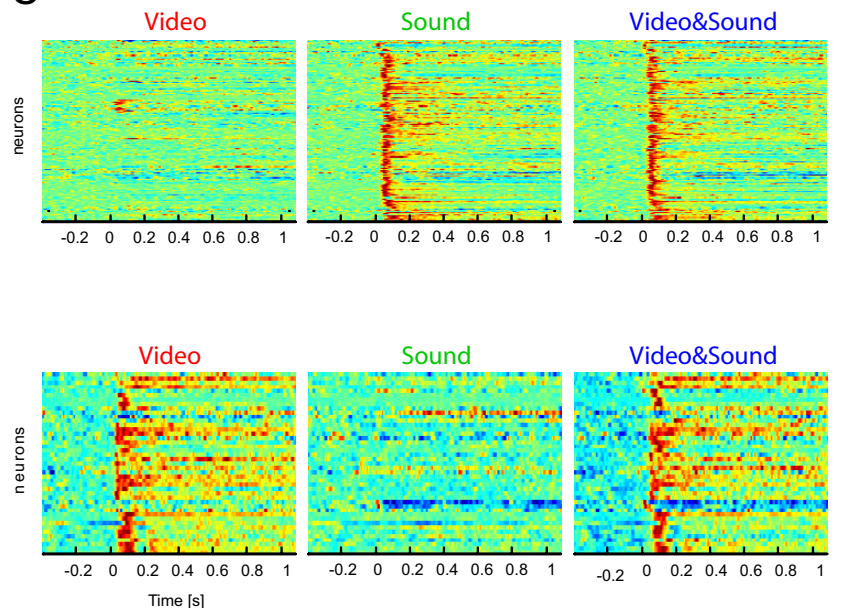

Figure 2. Unimodal responses in the auditory and visual zones. $\boldsymbol{A}$, Schematic of the claustrum with auditory (green) and visual (red) zones. $\boldsymbol{B}$, Population response within each zone to visual, auditory and combined audio-visual stimuli (median across neurons; auditory zone $n=148$; visual zone $n=43$ ). Neurons within the auditory zone responded to sounds and video and sound pairs, but not to the video alone. Neurons within the visual zone responded to videos but not to sounds. $C$, Responses of all units to each modality condition. Response amplitudes are color coded (red, high response) and have been normalized across neurons for display purposes. $\boldsymbol{D}$, Distribution of response amplitudes across units (boxplots indicate the median and $25^{\text {th }}$ and $75^{\text {th }}$ percentiles).

with sustained and quite variable responses, claustral responses to the same sounds were stereotyped and consisted of a strong transient followed by a minimal sustained response (supplemental Fig. 1, available at www.jneurosci.org as supplemental material). Third, during individual recording sessions the claustrum was distinguished from neighboring structures by the depth of the electrode and by observing response transitions between gray and white matter. All in all this allowed us to assign 191 neurons recorded along 157 electrode penetrations to the claustrum.

\section{Auditory and visually responsive zones}

Neural responses were recorded during an audio-visual paradigm consisting of the presentation of only the sound, only the video or the combined audio-visual stimulus while the animals performed a visual fixation task. Of the neurons assigned to the claustrum, some responded during the presentation of the video but not during the presentation of the sound, while some responded to the sound but not the video (Fig. $1 D$ ). One striking result emerging from our data is that these groups of neurons with visual and acoustic preferences were spatially separated. These results corroborate earlier reports of discrete sensory zones within the claustrum that are connected to the corresponding primary sensory cortical areas (Olson and Graybiel, 1980). In particular, a detailed analysis of our recordings revealed that in both animals auditory neurons were identified along the more dorsal electrode penetrations, while visual neurons were encountered at sites located more ventrally. This is illustrated in Figure $1 D$ for three vertical electrode tracks: along each track, neurons responding to the sound but not the video (upper examples) were encountered $\sim 20 \mathrm{~mm}$ from the cortical surface, while neurons responding to the video but not the sound (lower examples) were encountered a further $6 \mathrm{~mm}$ deeper. This spatial pattern of response preferences was consistent across electrode penetrations and animals. Our data hence highlight a region of neurons well driven by visual stimuli (termed visual zone) within the ventral claustrum and a region of neurons well driven by acoustic stimuli (auditory zone) dorsal to the visual zone and central within the claustrum (Fig. 1C,D).

Neurons within the auditory zone generally responded to sounds with a transient response followed by a smaller sustained response during the $1 \mathrm{~s}$ stimulus period (Fig. $2 \mathrm{~B}, \mathrm{C}$ ). The onset latency of this response was $40 \mathrm{~ms}$ (median value, $25^{\text {th }}$ and $75^{\text {th }}$ percentile: $35,60 \mathrm{~ms}$ ). The same neurons responded only minimally to visual stimuli and across neurons the response amplitude to visual stimuli was statistically indifferent from zero (Wilcoxon rank-sum test, $p \approx 0$; Fig. $2 D$ ). In addition, response amplitudes to auditory and bimodal audio-visual stimuli did not differ $(p=0.79)$. This dominance of auditory responses was further confirmed by directly comparing the responses to visual and auditory stimuli on a unit by unit basis (using a rank-sum test). This estimate of modality preference revealed that $86 \%$ of the units preferred sounds over videos, while $4 \%$ preferred videos over sounds and $10 \%$ did not show a significant preference (critical $p$-value, $p=0.05$; Fig. $3 A, B)$. The small fraction of not modality-selective units, together with the near-chance fraction of visual selective units clearly demarcates the "auditory" zone as dominated by acoustically driven responses and as region where neurons do not or minimally respond to visual stimuli.

Neurons within the visual zone responded to visual stimuli with a prominent onset response followed by a sustained elevation of firing during the rest of the video (Fig. $2 B, C$ ). The onset latency of this response was $55 \mathrm{~ms}$ (median value, $25^{\text {th }}$ and $75^{\text {th }}$ percentile: $45,87 \mathrm{~ms}$ ). The same neurons responded minimally to sounds and the average response amplitude to auditory stimuli was statistically indifferent from zero $(p=0$, Fig. $2 D)$. Response amplitudes to visual and bimodal audio-visual stimuli did not differ $(p=0.55)$. In addition, computing modality preferences showed that the vast majority of units responded preferentially to videos $(86 \%)$, while $12 \%$ did not show a significant preference and $2 \%$ preferred sounds over videos (Fig. $3 A, B$ ). Together, this demarcates the "visual" zone as visually driven region that does not respond to sounds.

To further verify the modality selectivity of the claustrum, we investigated local field potential (LFP) responses recorded on the same electrodes as the unit activity. In the auditory zone, response amplitudes to sounds were significantly higher than to videos (Wilcoxon rank-sum test, $p<10^{-3}$ ), while in the visual zone, response amplitudes were significantly higher for videos than sounds $\left(p<10^{-7}\right)$. 
A
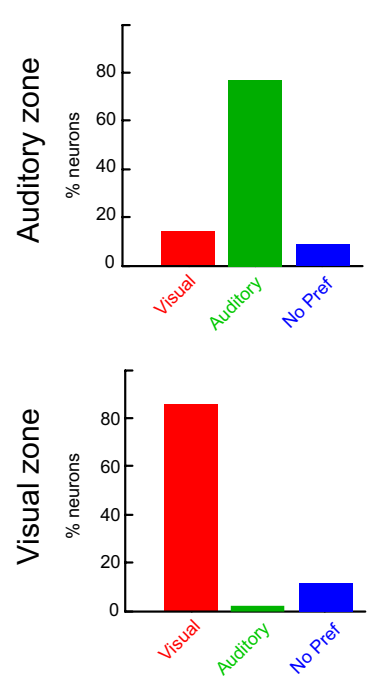

B
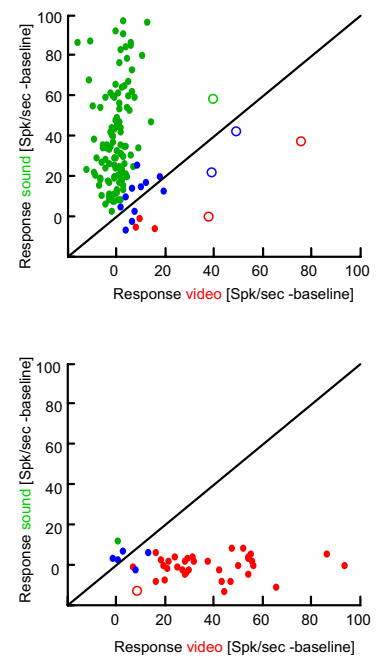

C
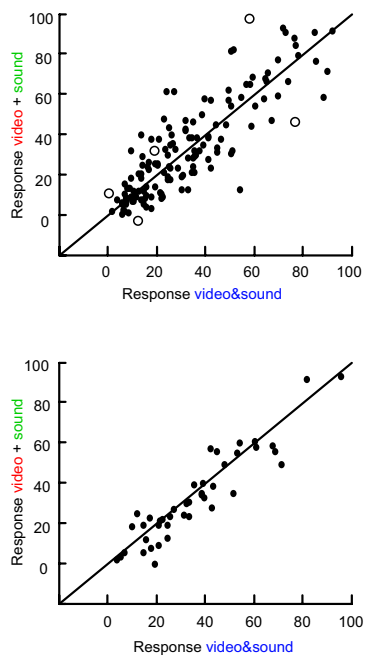

Figure 3. Modality preferences and lack of multisensory response interactions. $\boldsymbol{A}$, Modality preferences established by comparing responses to sounds and videos. $\boldsymbol{B}$, Response amplitudes to sounds and videos for individual units (dots). Units are color-coded according to their modality preference. Units depicted as circles were flagged as statistically significant bimodal, i.e., as exhibiting significant responses to both sensory modalities. C, Response amplitudes to audio-visual stimuli compared with the linear superposition of the responses to unimodal auditory and visual stimuli. A statistical bootstrap test revealed that only a negligible fraction of units (circles) exhibits statistically significant deviations from a linear superposition.

\section{Absence of functional signs of sensory integration}

The notions of multisensory processing and sensory integration can be formulated and tested at the level of neural responses using several criteria (Meredith and Stein, 1986; Stanford et al., 2005; Kayser and Logothetis, 2007; Stein and Stanford, 2008). Neurons are generally considered as unimodal (or bimodal in the case of two considered modalities) when they respond to stimuli in one sensory modality. Moreover, neurons are considered to be "integrating" stimuli from several modalities when the response to one sensory modality is significantly modulated by stimuli presented to another modality, i.e., when the responses to different sensory modalities interact.

Across both claustral zones we found that the two populations of neurons respond preferentially to their preferred modality and respond only minimally to stimuli in the other modality (cf. Fig. $2 D$ ), suggesting unimodal character. In addition we directly tested whether each unit in the visual or auditory zone also responded to sounds or videos respectively, with significant deviations of firing from the prestimulus baseline activity (critical $p$-value, $p=0.01$ ). This revealed that only $2 \%$ and $3 \%$ of the units in the visual and auditory zones were bimodal in the statistical sense (Fig. 3B, circles). This negligible fraction of significantly bimodal units demonstrates that the claustral neurons considered here can by and large be considered unimodal.

Consistently across both claustral zones we did not find response interactions between nonspecific stimuli presented to a particular modality. To establish this, we followed standard procedures and tested individual units for significant deviation from a model of linear response superposition: if a neuron is not affected by stimuli in a second modality, or trivially combines (sums) stimuli presented to different modalities, its response to the bimodal stimulus will be equal to the sum of the two unimodal stimuli presented in isolation. Significant deviations from such a linear response superposition can be detected using a bootstrap procedure (Stanford et al., 2005; Stein and Stanford, 2008). Of the neurons considered here, only $3 \%$ in the auditory zone and none in the visual zone exhibited a significant deviation from the linear model (critical $p$-value, $p=0.01$; circles in Fig. $3 C$ ). This insignificant fraction lets us conclude that the claustral units considered here do not show signs of multisensory processing or integration.

\section{Discussion}

Our results show that while the claustrum, as a structure, responds to stimuli in different sensory modalities, individual neurons show little evidence for multisensory processing. By recording neural responses to naturalistic audio-visual stimuli in the claustrum of awake behaving monkeys we were able to subdivide this structure into modality-specific zones. Neurons within a ventral zone were driven by visual stimuli while neurons within a more central zone were driven by sounds. Functional tests for multisensory influences revealed that neurons in both zones were insensitive to stimuli in their nonpreferred modality, highlighting a parcellation of the claustrum into distinct unimodal zones.

Early experiments on claustral function were aimed at detailing its anatomical differentiation (Narkiewicz, 1964) and revealed heterogeneous populations of neurons responding to stimuli in several sensory modalities (Spector et al., 1970, 1974). When electrophysiological recordings were used in parallel with neuroanatomical tracers, orderly and independent retinotopic and somatotopic maps were found within the cat's claustrum (Olson and Graybiel, 1980) and spatially patterned afferent projections from other sensory cortices were reported in the primate (Berke, 1960; Kemp and Powell, 1970; Künzle and Akert, 1977; Turner et al., 1980; Pearson et al., 1982; Ungerleider et al., 1984; Baizer et al., 1997; Tanné-Gariépy et al., 2002). To accommodate these widespread anatomical afferents and the heterogeneous neural response properties, the hypothesis regarding the claustrum as an integrator of sensory information was promoted (LeVay and Sherk, 1981; Sherk and LeVay, 1981; Ettlinger and Wilson, 1990; Edelstein and Denaro, 2004; Crick and Koch, 2005).

Support for an integrative role was also provided by noninvasive imaging studies that often revealed activations to multisensory stimuli (Ettlinger and Wilson, 1990; Hadjikhani and Roland, 1998; Banati et al., 2000; Naghavi et al., 2007) and sexually arousing stimuli (Redouté et al., 2000; Georgiadis et al., 2009) in the vicinity of the claustrum. However, given the claustrum's narrow width and the coarse spatial resolution offered by these techniques, it is often not possible to differentiate between activations originating from within the claustrum and those originating in adjacent regions, such as the insular cortex. As the insula integrates interoceptive representations and salient exteroceptive information to give rise to self and non-self awareness (Craig, 2009), multisensory responses here could be erroneously extrapolated to the adjacent claustrum. By directly recording neural responses in both these structures, we were able to disambiguate units to either the insula or claustrum on the basis of their response profiles (supplemental Fig. 1, available at www.jneurosci.org as supplemental material) (Remedios et al., 2009). Our results dem- 
onstrate the unimodal character of claustral neurons, and suggest that the multisensory aspects of claustral activation reported in imaging studies result either from spatially pooling activations originating in distinct structures, or from pooling activations across distinct claustral zones.

Despite directly probing neurons in the primate claustrum for functional criteria concerning multisensory processing, we found little evidence for integrative functions. Could it be that we missed essential multisensory responses in the claustrum? In our experiments we used naturalistic audio-visual stimuli, including videos and sounds of behaviorally relevant stimuli (e.g., the face of a conspecific vocalizing). If the claustrum were indeed to play a central role as a sensory integrator (Edelstein and Denaro, 2004; Crick and Koch, 2005), this would have been revealed while processing behaviorally relevant everyday scenes. However, it may be that any integrative function pertains to other modality combinations not tested here, e.g., involving the chemical senses, or only includes neurons or regions of the claustrum not sampled here. In addition, some aspects of claustral function may become apparent only in specific task contexts; as in comparing stimuli across modalities, estimating their congruency or amplifying the responses to subtle stimuli. While we cannot rule out any of these, it seems unlikely that we missed existing multisensory neurons along our electrode penetrations. We especially tried to sample every encountered neuron regardless of the quality of spike separation, and also included multiunit responses in our analysis, which actually biases our analysis in favor of detecting multisensory response properties. As the same stimulus set identified considerable fractions of bimodal or integrating neurons in the auditory cortices (Kayser et al., 2008) and the superior temporal sulcus (Dahl et al., 2010), this clearly suggests that integrating everyday sensory stimuli is unlikely to be the main function of the claustrum.

Our results suggest that the claustrum is not a simple relay for sensory information between cortical structures. For example, responses of neurons in the auditory zone to long naturalistic sounds consisted only of a brief transient following sound onset and did not reflect the full structure or duration of the sound (cf. Fig. 2). This response behavior clearly distinguishes claustral neurons from neurons in auditory related cortices that usually respond to the same sounds using sustained patterns of activity (see supplemental Fig. 1, available at www.jneurosci.org as supplemental material and (Kayser et al., 2008; Remedios et al., 2009). As a result, claustral activity seems to represent the occurrence of a sound rather than to its detailed acoustical properties. Noteworthy, response latencies within the claustrum were comparatively short and on the order of those reported for other early sensory cortices. This suggests that the major driving input to the claustrum originates in thalamic structures and early sensory cortices, providing the claustrum with rapid information about the occurrence of external events. Together, this can be taken as evidence for a role in detecting sudden or important changes within an animal's external environment, akin to a saliency detector. By virtue of its widespread connectivity with the cortex (Sherk, 1986) the claustrum could send such alerting or awareness signals to several cortical areas at the same time.

Our results underscore the need for rethinking the function of the claustrum, which, despite much speculation and interest, still remains elusive. While our results demonstrate that the claustrum as structure has access to different sensory modalities, our results challenge the notion that it integrates information across these modalities. While offering new directions for future work, we assent that much needs to be done to unravel the functions of this enigmatic structure.

\section{References}

Baizer JS, Lock TM, Youakim M (1997) Projections from the claustrum to the prelunate gyrus in the monkey. Exp Brain Res 113:564-568.

Banati RB, Goerres GW, Tjoa C, Aggleton JP, Grasby P (2000) The functional anatomy of visual-tactile integration in man: a study using positron emission tomography. Neuropsychologia 38:115-124.

Berke JJ (1960) The claustrum, the external capsule and the extreme capsule of Macaca mulatta. J Comp Neurol 115:297-331.

Clarey JC, Irvine DR (1986) Auditory response properties of neurons in the claustrum and putamen of the cat. Exp Brain Res 61:432-437.

Cortimiglia R, Crescimanno G, Salerno MT, Amato G (1991) The role of the claustrum in the bilateral control of frontal oculomotor neurons in the cat. Exp Brain Res 84:471-477.

Craig AD (2009) How do you feel—now? The anterior insula and human awareness. Nat Rev Neurosci 10:59-70.

Crick FC, Koch C (2005) What is the function of the claustrum? Philos Trans R Soc Lond B Biol Sci 360:1271-1279.

Dahl CD, Logothetis NK, Kayser C (2009) Spatial organization of multisensory responses in temporal association cortex. J Neurosci 29:1192411932.

Dahl CD, Logothetis NK, Kayser C (2010) Modulation of visual responses in the superior temporal sulcus by audio-visual congruency. Front Integr Neurosci 4:10.

Edelstein LR, Denaro FJ (2004) The claustrum: a historical review of its anatomy, physiology, cytochemistry and functional significance. Cell Mol Biol (Noisy-le-grand) 50:675-702.

Ettlinger G, Wilson WA (1990) Cross-modal performance: behavioural processes, phylogenetic considerations and neural mechanisms. Behav Brain Res 40:169-192.

Fernández-Miranda JC, Rhoton AL Jr, Kakizawa Y, Choi C, Alvarez-Linera J (2008) The claustrum and its projection system in the human brain: a microsurgical and tractographic anatomical study. J Neurosurg 108:764-774.

Georgiadis JR, Simone Reinders AA, Paans AM, Renken R, Kortekaas R (2009) Men versus women on sexual brain function: prominent differences during tactile genital stimulation, but not during orgasm. Hum Brain Mapp 30:3089-3101.

Hadjikhani N, Roland PE (1998) Cross-modal transfer of information between the tactile and the visual representations in the human brain: a positron emission tomographic study. J Neurosci 18:1072-1084.

Kayser C, Logothetis NK (2007) Do early sensory cortices integrate crossmodal information? Brain Struct Funct 212:121-132.

Kayser C, Petkov CI, Logothetis NK (2008) Visual modulation of neurons in auditory cortex. Cereb Cortex 18:1560-1574.

Kemp JM, Powell TP (1970) The corticostriate projection in the monkey. Brain 93:525-546.

Künzle H, Akert K (1977) Efferent connections of cortical area 8 (frontal eye field) in Macaca fascicularis. A reinvestigation using the autoradiographic technique. J Comp Neurol 173:147-164.

LeVay S, Sherk H (1981) The visual claustrum of the cat. II. The visual field map. J Neurosci 1:981-992.

Meredith MA, Stein BE (1986) Visual, auditory, and somatosensory convergence on cells in superior colliculus results in multisensory integration. J Neurophysiol 56:640-662.

Naghavi HR, Eriksson J, Larsson A, Nyberg L (2007) The claustrum/insula region integrates conceptually related sounds and pictures. Neurosci Lett 422:77-80.

Narkiewicz O (1964) Degenerations in the claustrum after regional neocortical ablations in the cat. J Comp Neurol 123:335-355.

Olson CR, Graybiel AM (1980) Sensory maps in the claustrum of the cat. Nature 288:479-481.

Pearson RC, Brodal P, Gatter KC, Powell TP (1982) The organization of the connections between the cortex and the claustrum in the monkey. Brain Res 234:435-441.

Redouté J, Stoléru S, Grégoire MC, Costes N, Cinotti L, Lavenne F, Le Bars D, Forest MG, Pujol JF (2000) Brain processing of visual sexual stimuli in human males. Hum Brain Mapp 11:162-177.

Remedios R, Logothetis NK, Kayser C (2009) An auditory region in the primate insular cortex responding preferentially to vocal communication sounds. J Neurosci 29:1034-1045. 
Segundo JP, Machne X (1955) Unitary responses to afferent volleys in lenticular nucleus and claustrum. J Neurophysiol 19:325-339.

Sherk H, ed (1986) The claustrum and the cerebral cortex. New York: Plenum.

Sherk H, LeVay S (1981) Visual claustrum: topography and receptive field properties in the cat. Science 212:87-89.

Spector I, Hassmannova Y, Albe-Fessard D (1970) A macrophysiological study of functional organization of the claustrum. Exp Neurol 29:31-51.

Spector I, Hassmannova Y, Albe-Fessard D (1974) Sensory properties of single neurons of cat's claustrum. Brain Res 66:39-65.

Stanford TR, Quessy S, Stein BE (2005) Evaluating the operations underlying multisensory integration in the cat superior colliculus. J Neurosci 25:6499-6508.
Stein BE, Meredith MA (1993) Merging of the senses. Cambridge, MA: MIT.

Stein BE, Stanford TR (2008) Multisensory integration: current issues from the perspective of the single neuron. Nat Rev Neurosci 9:255-266.

Tanné-Gariépy J, Boussaoud D, Rouiller EM (2002) Projections of the claustrum to the primary motor, premotor, and prefrontal cortices in the macaque monkey. J Comp Neurol 454:140-157.

Turner BH, Mishkin M, Knapp M (1980) Organization of the amygdalopetal projections from modality-specific cortical association areas in the monkey. J Comp Neurol 191:515-543.

Ungerleider LG, Desimone R, Galkin TW, Mishkin M (1984) Subcortical projections of area MT in the macaque. J Comp Neurol 223:368-386. 